\title{
The ABA-induced soybean ERF transcription factor gene GmERF75 plays a role in enhancing osmotic stress tolerance in Arabidopsis and soybean
}

\author{
Meng-Jie Zhao ${ }^{1 \dagger}$, Li-Juan Yin ${ }^{1 \dagger}$, Ying Liu ${ }^{1 \dagger}$, Jian Ma², Jia-Cheng Zheng ${ }^{3}$, Jin-Hao Lan", Jin-Dong Fu', Ming Chen',
} Zhao-Shi $\mathrm{Xu}^{1,2,3^{*}}$ and You-Zhi Ma'

\begin{abstract}
Background: Ethylene-responsive factors (ERFs) play important roles in plant growth and development and the response to adverse environmental factors, including abiotic and biotic stresses.

Results: In the present study, we identified 160 soybean ERF genes distributed across 20 chromosomes that could be clustered into eight groups based on phylogenetic relationships. A highly ABA-responsive ERF gene, GmERF75, belonging to Group VII was further characterized. Subcellular localization analysis showed that the GmERF75 protein is localized in the nucleus, and qRT-PCR results showed that GmERF75 is responsive to multiple abiotic stresses and exogenous hormones. GmERF75-overexpressing Arabidopsis lines showed higher chlorophyll content compared to WT and mutants under osmotic stress. Two independent Arabidopsis mutations of AtERF71, a gene homologous to GmERF75, displayed shorter hypocotyls, and overexpression of GmERF75 in these mutants could rescue the short hypocotyl phenotypes. Overexpressing GmERF75 in soybean hairy roots improved root growth under exogenous ABA and salt stress.

Conclusions: These results suggested that GmERF75 is an important plant transcription factor that plays a critical role in enhancing osmotic tolerance in both Arabidopsis and soybean.
\end{abstract}

Keywords: Ethylene-responsive factor, Hypocotyl elongation, Root growth, Response mechanism, Osmotic tolerance, Soybean

\section{Background}

Plants have a complex and elaborate regulation mechanism to defense the environmental factors including abiotic and biotic stresses $[1,2]$. Transcription factors, regulators of genes expression, perform pivotal functions in signal transduction networks where they directly activate or suppress targeted genes expression so that the interaction between different signaling pathways was impacted [3-5].

\footnotetext{
*Correspondence: xuzhaoshi@caas.cn

${ }^{+}$Meng-Jie Zhao, Li-Juan Yin and Ying Liu contributed equally to this work. ${ }^{1}$ Institute of Crop Sciences, Chinese Academy of Agricultural Sciences (CAAS)/National Key Facility for Crop Gene Resources and Genetic Improvement, Key Laboratory of Biology and Genetic Improvement of Triticeae Crops, Ministry of Agriculture, Beijing 100081, China

${ }^{2}$ Faculty of Agronomy, Jilin Agricultural University, Changchun 130118, China Full list of author information is available at the end of the article
}

APETALA2/Ethylene Responsive Factor (AP2/ERF) superfamily, a large gene family in plant, play important roles in signal transduction, plant growth and development, and involved in biotic and abiotic stresses response [6]. According to its conservative domain, AP2/ERF can be divided into three major families: APETALA2(AP2), Ethylene Responsive Factor (ERF), and RELATED TO ABSCISIC ACID INSENSITIVE 3/VIVIPAROUS 1 (RAV) [7]. The AP2 family contains two AP2/ERF domain, ERF family which can be divided into two subfamilies: DEHYDRATION-RESPONSIVE ELEMENT BINDING proteins (DREBs) and ERFs, contains an AP2/ERF domain, and RAV family contains an AP2/ERF domain [7]. ERFs play diverse roles in plants throughout different development stage, such as seed germination, tissue formation, flower stage, response to biotic and abiotic

(C) The Author(s). 2019 Open Access This article is distributed under the terms of the Creative Commons Attribution 4.0 International License (http://creativecommons.org/licenses/by/4.0/), which permits unrestricted use, distribution, and 
stresses $[8,9]$. Previous study found that ERFs could specifically bind to the GCC-box and/or dehydration-responsive element/C-repeat (DRE/CRT) cis-acting elements to regulate the downstream gene expression, such as ethylene (ET)-inducible pathogenesis-related (PR) genes and abiotic stresses-inducible genes [10]. .In recent years, it was found that ERFs could also bind to Coupling Element 1 (CE1: TGCCACCG), Hypoxia-Responsive Promoter Element (HRPE), and ATCTA [11, 12]. ERFs, were first identified in tobacco, since then more and more ERFs have been identified in diverse plants, including Arabidopsis, rice, Atriplex canescens, peanuts, sunflower, and potato [7, 13-18].

ERF could influence the growth and development in plant. The activity of some ERFs was impacted by different development stage [19]. Overexpressing LkAP2L2 in Arabidopsis, which could affect seed growth, branch, flower development, and siliques, significantly enhanced the number of shoot branches and decreased the length of siliques, the number of seeds, the size and number of transgenic rosette leaves [8]. OsHL6, an AP2/ERF transcript factor in rice, could regulate the expression of some auxin-related genes by interacting with OsWOX3 and play critical roles in trichome formation [9].

ERF genes can also function in abiotic and/or biotic stress responsive pathways. TaERF1, a wheat ERF gene which could be induced by multiple environmental stresses including drought, salt, low temperature, and exogenous hormones such as ABA, ET, and salicylic acid (SA), was also identified as a defense gene against pathogen (Blumeria graminis f. sp. tritici). Overexpression of TaERF1 in Arabidopsis and tobacco could improve resistance to pathogens and enhance tolerance to multiple abiotic stresses [20]. Haynaldia villosa ERF1-V regulated the response to both powdery mildew and drought and salinity when overexpressed in wheat [21]. Similarly, TaPIE1, a member of ERF family in wheat, enhanced resistance to Rhizoctonia cerealis and increased tolerance to freezing stress by activating defense- and stress-related genes that function downstream of the ET signaling pathway in wheat [22]. Therefore, ERF genes could encode multifunctional factors that respond to multiple stresses, integrate potentially various signal transduction pathways, and thus play dual roles in both abiotic and biotic stress responses in plants [14, 23].

Although ERFs have been found in diverse plants, many soybean ERFs have not been reported yet, which is one of the most economically important crop species. In addition, the functions of most ERF genes have yet to be determined. In this study, we searched for and integrated all nonredundant sets of soybean ERF genes. GmERF75, a highly ABA-induced ERF gene, was chosen for further expression and functional analysis. GmERF75 was up-regulated by multiple abiotic stresses and exogenous hormones, and overexpression of which could enhance osmotic tolerance in both Arabidopsis and soybean.

\section{Results}

Identification and physical locations of soybean ERFs We used the Pfam [24] and SMART databases [25] as references for the identification of 160 non-redundant soybean ERFs (Additional file 1: Table S1). According to the soybean genome database, 160 soybean ERFs were distributed across 20 chromosomes (Fig. 1). The number of ERF genes on each chromosome differed considerably. There were 17 ERF genes distributed in chromosome 13, but only 3 in chromosome 12 (Fig. 1). Multiple alignments of full-length amino acid sequences were performed using MEGA 5.1 [26] . The ERF proteins could be clustered into eight groups (I to VIII) based on their phylogenetic relationships (Fig. 2). Almost one-fourth of the ERF proteins were clustered in Group I, while only nine were clustered in Group IV.

\section{Expression profiles of soybean ERFs}

To examine the expression patterns of ERFs, a map of soybean ERF gene expression in 14 soybean tissues and organs at different developmental stages was drawn based on the gene-chip data downloaded from the soybean genome database (Additional file 2: Figure S1; Additional file 3: Table S2). Soybean ERFs were expressed at the highest levels in the nodules of 21 days-old plants and at the lowest levels in seeds. A few soybean ERFs displayed different tissue-specific expression patterns. For example, eight ERFs were expressed in only one tissue, and nine ERFs were expressed in only two tissues. The expression levels for genes in different groups also differed. The expression levels of Group II genes were lower than those of genes in the other groups. The expression patterns of ERFs within the same group also varied. For example, GmERF127 transcripts reached the maximum level in flowers, whereas GmERF10 transcripts reached the highest level in roots. GmERF6, GmERF66, and GmERF84 were expressed at a low level, whereas GmERF52, GmERF112, GmERF122, and GmERF124 were expressed at an extremely high level. Interestingly, three-quarters of the extremely high-expressed ERF genes were clustered in Group VII. Therefore, Group VII was selected for further investigation.

\section{Conserved protein motifs and gene structures of soybean group VII ERFs}

There are 12 ERF genes belonging to Group VII. To investigate the modular structure of the proteins encoded by these genes, DOG 2.0 was used to draw the domains in each protein. As shown in Additional file 4: Figure S2, each Group VII ERF protein had a typical AP2/ERF DNA-binding domain, which is highly conserved, consists of 57-61 amino acids, and contains three $\beta$-sheet regions and an $\alpha$-helix. The key amino acid residues determining 

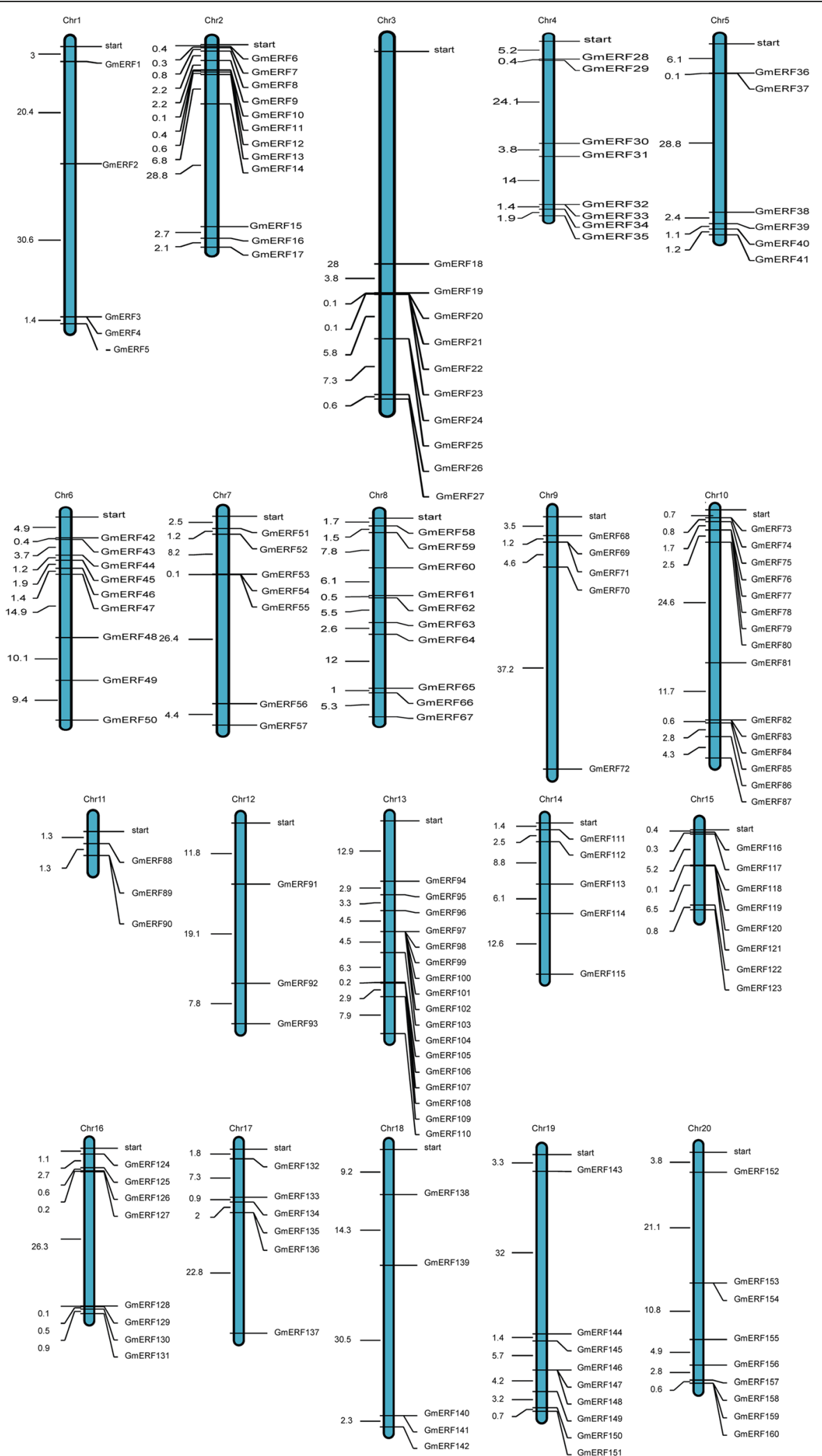

Fig. 1 Distribution of ERF genes in the soybean genome. The blue bars represent the chromosomes (not drawn to scale), and the chromosome numbers are shown above the bars. Soybean ERFs were distributed on all 20 chromosomes. The numbers to the left of the chromosomes indicate the distances between the neighboring genes in megabases (Mb) 


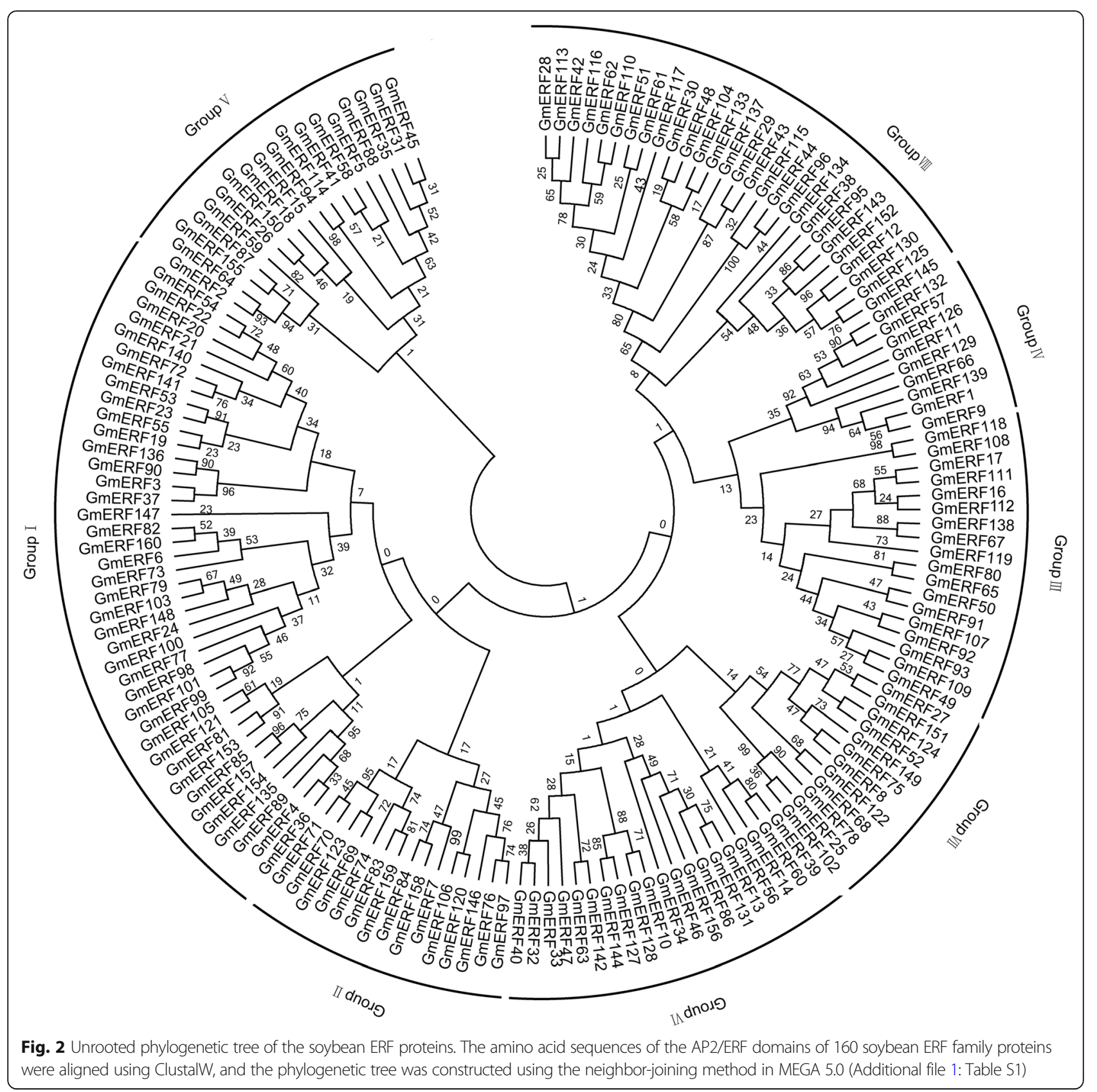

DNA-binding specificity are those at positions 14, Ala (A) and 19, Asp (D) [10].

Gene structure analysis was done to compare the distribution of introns and exons in each soybean ERF gene. Almost all the ERF genes contained one intron except for GmERF102, GmERF25, and GmERF78 which contained no intron (Additional file 5: Figure S3).

\section{Expression pattern of GmERF75 under ABA treatment}

ABA plays essential role in regulating seed germination, growth and development, and responses to environmental stresses in plants $[10,27,28]$. It has been reported that most drought-inducible and/or salt-inducible genes were also induced by exogenous ABA treatment in Arabidopsis [29], which suggested the existence of cross-talk between $\mathrm{ABA}$ and osmotic stress signaling pathways.

To investigate the expression levels of the 12 soybean ERFs after ABA treatment, quantitative real-time PCR (qRT-PCR) was conducted using cDNA obtained from hypocotyls and roots of ABA-treated soybean seedlings as a template. As shown in Fig. 3, almost all soybean ERFs were up-regulated to different extents in response to exogenous ABA treatment (Figs. 3a-l). Transcription level of GmERF75 was the highest up-regulated and 


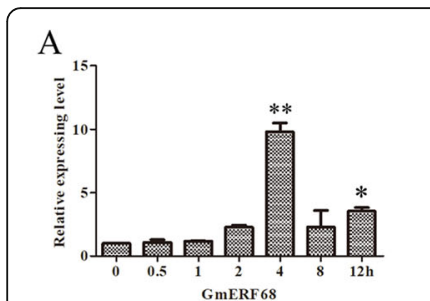

E

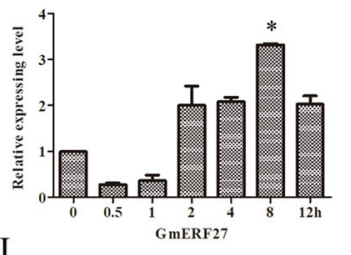

I

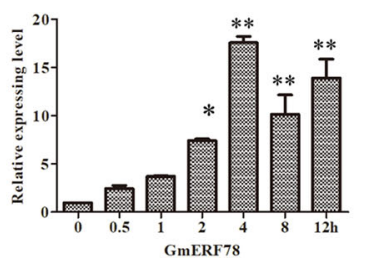

B

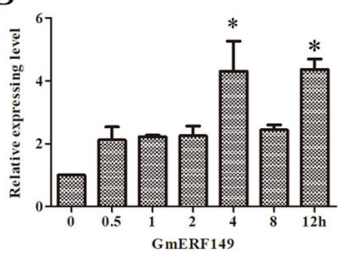

F

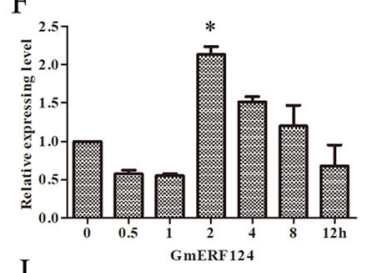

J

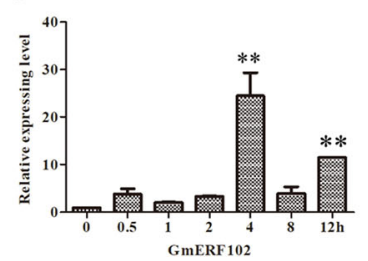

C

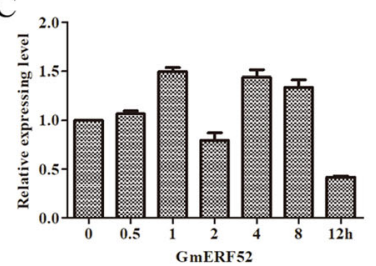

G
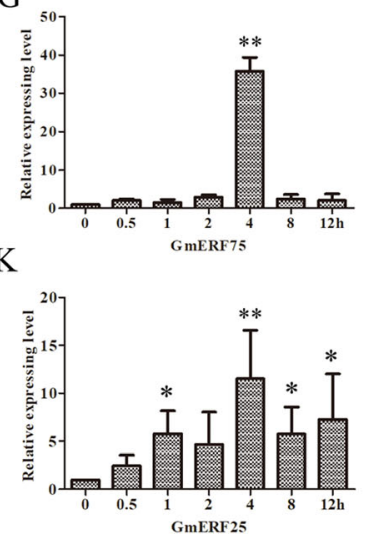

D

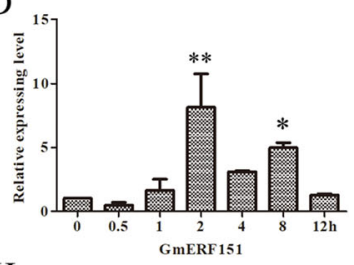

$\mathrm{H}$

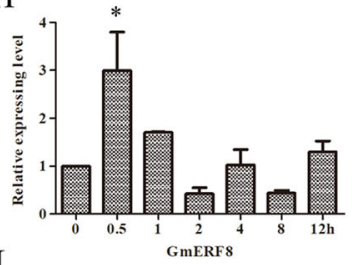

L

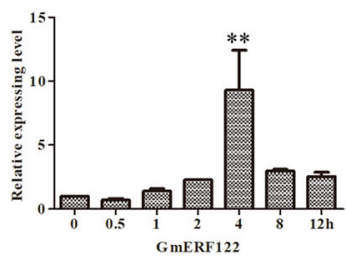

Fig. 3 ABA-induced Group VII ERF genes expression. 14-day-old soybean seedlings were treated with ABA and collected the hypocotyl and roots $0,0.5,1,2,4,8,12 \mathrm{~h}$ after treatment for RNA extraction and qRT-PCR. Expression levels of the 12 Group VII ERFs in response to ABA treatment were revealed by $\mathrm{qRT}-\mathrm{PCR}$. The data was shown as the means \pm SD of three biological replicates

reached the highest level at $4 \mathrm{~h}$ after ABA treatment (Fig. $3 g)$. Therefore, GmERF75 was selected for further study.

To investigate the expression pattern of GmERF75 in different soybean plant tissues, semi-quantitative PCR (semi-qPCR) was conducted. RNA was extracted from hypocotyls, roots, stems, and leaves of soybean seedlings. Parallel reactions amplifying Actin were performed to normalize the expression levels. This result showed that GmERF75 is predominantly expressed in hypocotyls and roots, with less expression observed in leaves (Additional file 6: Figure S4).

\section{GmERF75 is localized in nucleus}

The CDS of GmERF75 was acquired that contained complete 903 bp open reading frame (ORF), which encodes a putative protein of 300 amino acids (Additional file 7: Figure S5). The GmERF75 protein contains a putative basic amino acid region (KPVKRQRK) that potentially act as a nuclear localization sequence (NLS), and acidic amino acid regions, EKETEVIEAEEEKNKVLELSEE and EEEEVVVEE, in the C-terminal region that may act as transcriptional activation domains (Additional file 7: Figure S5).

To investigate whether the GmERF75 protein located in cell nucleus, the full-length ORF of GmERF75 was amplificated and fused in frame with the hGFP gene under the control of the CaMV $35 \mathrm{~S}$ promoter and then transferred into onion epidermal cells to observe fluorescence signal (Fig. 4). The result showed that GmERF75::hGFP fusion protein fluorescence was predominantly observed in the nucleus. GFP fluorescence of the control one was distributed throughout the cell. These results indicated that the GmERF75 fusion protein was targeted to nucleus.

\section{The GmERF75 promoter region contains diverse stress- responsive elements}

To further investigate the transcriptional regulation of GmERF75, 1809 bp promoter region of GmERF75 upstream of the ATG start codon was isolated. Putative cis-acting elements in the promoter region were identified using the PLACE (http://www.dna.affrc.go.jp/ PLACE/) and PlantCARE (http://bioinformatics.psb. ugent.be/webtools/plantcare) databases. Several distinct regulatory motifs homologous to cis-acting elements involved in responses to abiotic and biotic stresses and plant hormones were identified (Table 1).

Many abiotic and biotic stress-related cis-elements are distributed in the promoter region of GmERF75. There are eight hormone-responsive elements including five ABA relative elements (i.e., an AERB, two MYBST1 core binding site sequences, a DPBF binding site, and a MYB binding site), a gibberellic acid responsive element (GARE), a SA responsive element (TCA element), and an auxin responsive element (TGA element). Pathogen related elements (a W-box, and a TC-rich repeat) were also found in the promoter region. Interestingly, the RE $\alpha$ element (AACCAA), which is highly bound in etiolated plants but lowly bound in green plants, was found in the GmERF75 promoter region (Table 1). In addition, a 


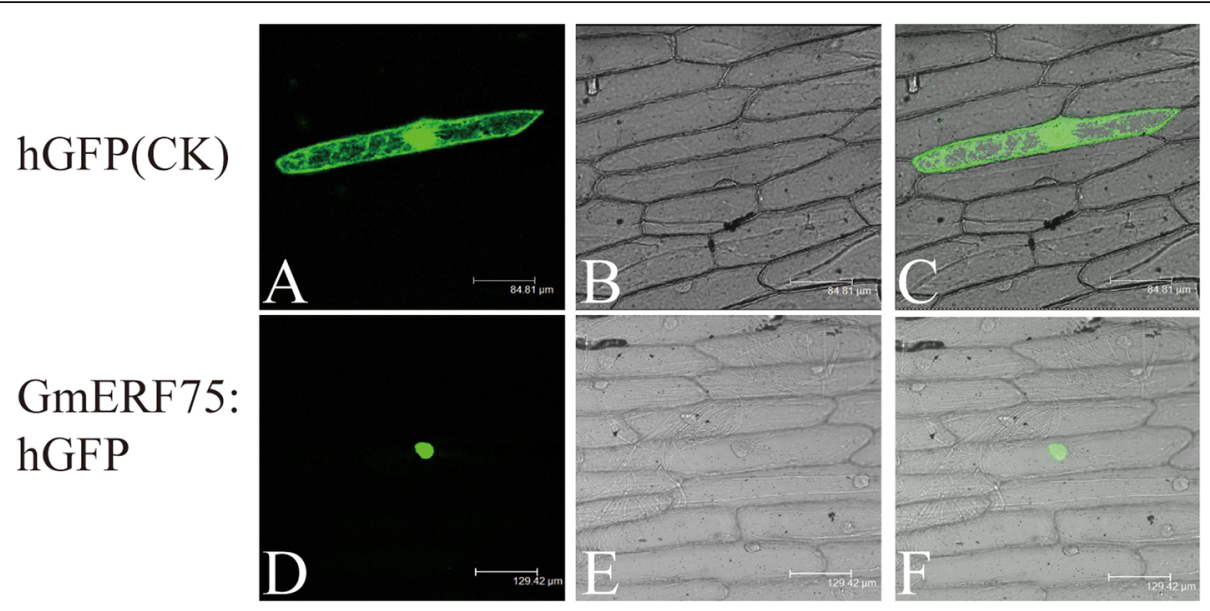

Fig. 4 Subcellular localization of the GmERF75 protein. GmERF75-hGFP vector and control $h$ GFP vector were bombarded into living onion epidermal cells. Localization of GmERF75 in onion epidermal cells was investigated using a confocal microscope (Leica). Photographs were taken in dark field to visualize green fluorescence (a and $\mathbf{d}$ ) and in bright light to visualize cell morphology (b and $\mathbf{e}$ ). Merged dark field and bright light images are shown in (c and $\mathbf{f}$ ). Scale bars were shown in the bottom of each photo

series of light-responsive elements such as Box-4, G-box, ACE, and ACGT-element were also found in the GmERF75 promoter region (Table 1). The presence of these cis-acting elements suggested that the expression level of GmERF75 could be regulated by multiple stresses, which in turn indicated that GmERF75 may participate in several signal transduction pathways.

\section{Changes in GmERF75 expression in response to abiotic stresses and exogenous hormones}

To investigate the expression level of GmERF75 under abiotic stresses including drought, salt, and high/low temperature, and in the presence of exogenous hormones, qRT-PCR was conducted using total RNA extracted from hypocotyls and roots of soybean seedlings

Table 1 Analysis of putative cis-acting elements in the GmERF75 promoter

\begin{tabular}{llll}
\hline GmERF75 & Cis-acting elements & Core sequences & Functions \\
\hline+ & W-box & TTGACC & fungal elicitor responsive element \\
+ & ACGT-element & ACGT & dehydration and dark-induced senescence \\
+ & core of MYBST1 & GGATA & ABA and stress responsive element \\
+ & core of MYBST1 & GGATA & ABA and stress responsive element \\
+ & ABRE & ACGTG & ABA responsive element \\
+ & DPBF binding site & ACACNNG & ABA responsive element \\
+ & GARE & TAACAAR & gibberellin responsive element, \\
+ & CAAT-box & CCAATT & common element in enhancer region \\
+ & Box-4 & ATTAAT & light responsive element \\
+ & G-box & CACGAC & light responsive element \\
+ & G-box & light responsive element \\
+ & ACE & CACGTG/T & light responsive element \\
+ & ACE & AAAACGTTA & light responsive element \\
+ & TCA element & CTAACGTAT & salicylic acid responsive element \\
+ & TGA element & GAGAAGATA & auxin responsive element \\
+ & TC-rich repeat & AACGAC & defense and stress responsive element \\
+ & MYB binding site & ATTCTCTAAC & ABA and stress responsive element \\
\hline & REa element & WAACCA & DNA binding activity is high in etiolated plants
\end{tabular}


as a template. All of the treatments increased the expression level of GmERF75, particularly ET (about 75-fold increase). As shown in Fig. 5, GmERF75 was rapidly induced by ET, exhibiting the highest increase in expression which has a 75 -fold change within $1 \mathrm{~h}$ after ET treatment, and then expression gradually declined to normal level observed before treatment. Upon high temperature treatment, GmERF75 expression peaked at $12 \mathrm{~h}$ (about 18-fold) and then declined to the initial level within $24 \mathrm{~h}$ (Fig. 5). Transcription of GmERF75 was also up-regulated by drought (about 6-fold) and salt treatment (about 4-fold), and for both treatments expression levels were the highest at $0.5 \mathrm{~h}$ and declined to initial level within $24 \mathrm{~h}$. Low temperature could increase GmERF75 transcription level by 4 times after $2 \mathrm{~h}$ of treatment. Expression levels also increased in response to exogenous SA. These results suggest that GmERF75 may play a crucial role in numerous signal transduction pathways related to stress [30].

\section{GmERF75 overexpression rescued two Arabidopsis erf71 mutants hypocotyl elongation}

To investigate the function of GmERF75 in Arabidopsis, AtERF71 was identified as a homologous gene of GmERF75, which share $55.47 \%$ identity compare to GmERF75. Two Arabidopsis erf71 mutants (SALK_ 030459C, CS362782) were found to display shorter roots and hypocotyls compared with wild-type (WT) Arabidopsis [31] (Additional file 8: Figure S6). To assess whether GmERF75 could rescue the phenotype of erf71 mutants, GmERF75 was introduced into the two mutants under the control of the CaMV $35 \mathrm{~S}$ promoter, and transgenic GmERF75::erf71 lines were obtained. T3 seeds of stable genetically inherited plants were used for further phenotypic analysis. Significant differences between WT and erf71 mutants hypocotyl length were observed. The erf71 mutants displayed shorter hypocotyls, while the GmERF75:: erf71 lines shared the similar phenotype with WT (Additional file 8: Figure S6). This result indicated that GmERF75 could promote hypocotyl growth.

\section{GmERF75 improved osmotic stress tolerance in transgenic Arabidopsis plants}

The GmERF75 gene was strongly induced by various abiotic stresses (Fig. 5). To evaluate the contribution of the GmERF75 gene to abiotic stress tolerance, two GmERF75-overexpressing Arabidopsis lines were grown under PEG, $\mathrm{NaCl}$, and dark conditions. The GmERF75-overexpressing lines displayed longer hypocotyls under different abiotic stresses than WT Arabidopsis plants (Fig. 6a). The largest differences in hypocotyl length between the 35S::GmERF75 lines and WT were observed after 5 days of treatment with 75 mM salt and 6\% PEG (Fig. 6c).

To test the tolerance to salt and drought in late stage of Arabidopsis, three-week-old seedlings were treated with $250 \mathrm{mM} \mathrm{NaCl}$ for 2 weeks or not watered for 1 week and then re-watered (Fig. 6b). The chlorophyll content of each line were recorded (Fig. 6d). The result showed that the chlorophyll content of transgenic plants under salt treatment was increased by 20.11 and $39.66 \%$ compared to WT and the mutants, respectively. For drought treatment, the chlorophyll content of transgenic plants was increased by $29.70 \%$ compared to WT, Taken together, these results suggest that GmERF75 has a role in improving tolerance to osmotic stress in Arabidopsis.

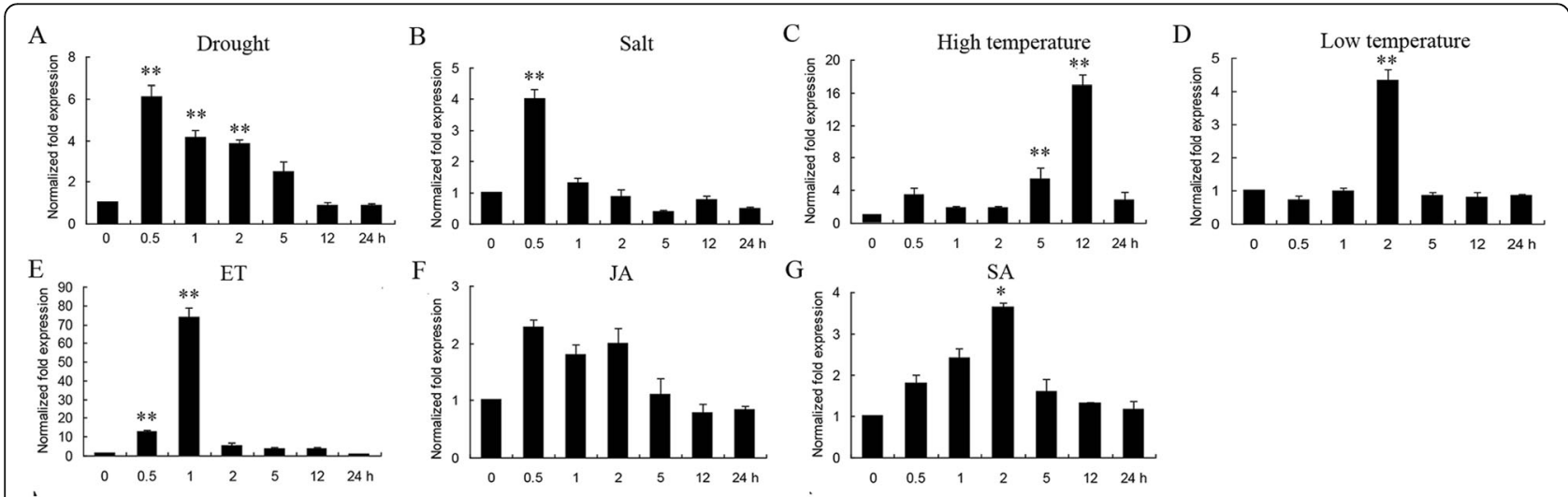

Fig. 5 Changes in GmERF75 expression in response to abiotic stress treatments and exogenous hormones. The kinetics of GmERF75 mRNA accumulation were evaluated for hypocotyl and root of 14-day-old seedlings subjected to the abiotic stress treatments drought (a), NaCl (b), high temperature (c), and low temperature (d), or treated with the exogenous hormones ethylene (ET, e), jasmonate (JA, $\mathbf{f}$ ), and salicylic acid (SA, g). The total RNA was extracted $0,0.5,1,2,5,12$, and $24 \mathrm{~h}$ after each treatment and used for qRT-PCR. The data was shown as the means \pm SD of three biological replicates 

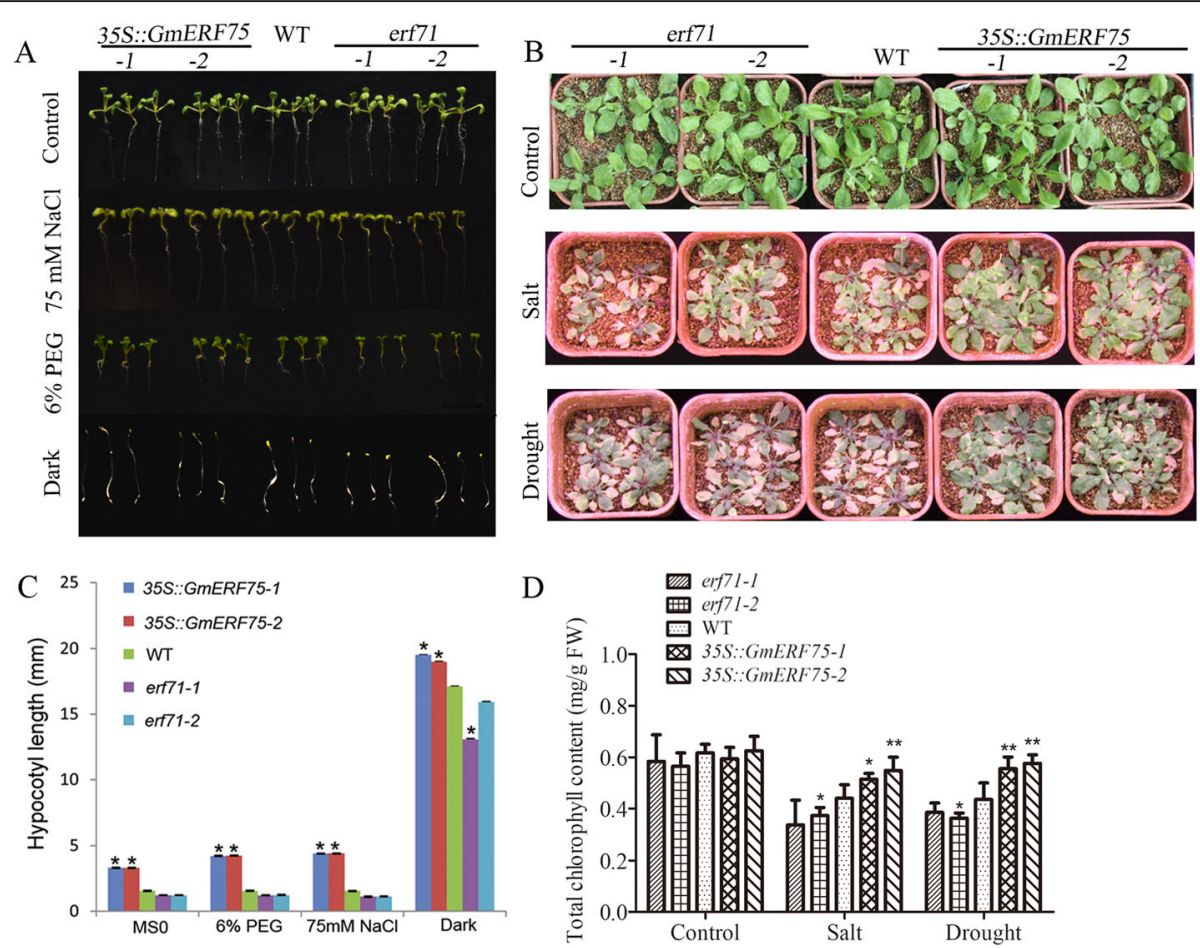

Fig. 6 Overexpression of GmERF75 in Arabidopsis enhanced tolerance of osmotic stress. a GmERF75-overexpressing lines had longer hypocotyls than the WT and the two erf71 mutants. Arabidopsis seedlings of GmERF75-overexpressing, WT, and mutants grown on MS medium with/without 6\% PEG, $75 \mathrm{mM} \mathrm{NaCl}$, or dark condition, respectively. b Overexpression of GmERF75 in Arabidopsis enhance the resistance to salt and drought. Three-week-old seedlings normally grown in soil were supplied with $250 \mathrm{mM} \mathrm{NaCl}$ for 2 weeks or were not watered for 1 week and then were re-watered for 1 week. c Hypocotyl length of 355::GmERF75, erf71, and WT. Fifteen to twenty-five individuals in each treatment were used to count the hypocotyl length. The data was shown as the means \pm SD of three biological replicates. $\mathbf{d}$ Total chlorophyll content of the mutants, WT (Col-0), and GmERF75 transgenic lines under drought and salt treatment. Three-week-old seedlings of mutants, WT (Col-0), and transgenic lines were supplemented with $250 \mathrm{mM} \mathrm{NaCl}$ or un-watered for 1 week, and recovered for 1 week. $0.1 \mathrm{~g}$ leaves of each line were collected and used to measure chlorophyll content. The data was shown as the means \pm SD of five repetitions each lines. Asterisks indicate significant differences from $W T$ at ${ }^{*} P<0.05$ and ${ }^{*} P<0.01$ determined by Student's $t$ test

\section{GmERF75 improved tolerance to salt stress and exogenous ABA in transgenic soybean hairy roots} To further investigate the function of GmERF75 in stress tolerance in soybean, a $p$ GFPGUSPlus vector designed to express $p$ GFPGUSPlus-GmERF75 was constructed and then transformed into Cucumopine-type Agrobacterium rhizogene strain K599, which was injected into Superroot of Lotus corniculatus. The positive transgenic hairy roots cultured on 1/2 Murashige and Skoog (MS) medium containing $\mathrm{PEG}, \mathrm{NaCl}$, or $\mathrm{ABA}$, which were verified via GFP fluorescence. Transgenic hairy roots were much longer than vector control hairy roots under $\mathrm{NaCl}$ treatment in seedling stage (Fig. 7a). The higher dry weights of transgenic hairy roots also supported this conclusion (Fig. 7b). As shown in Fig. 7b, transgenic hairy roots transformed with $p$ GFPGUSPlus-GmERF75 exhibited more growth than those transformed with the empty vector control under different concentrations of $\mathrm{NaCl}$ and ABA. Extremely significant differences between the transgenic and control hairy roots were observed under 85 and $120 \mathrm{mM} \mathrm{NaCl}$ treatment, and significant differences were also observed under 50 and $100 \mu \mathrm{M}$ ABA. However, there was no obvious difference between transgenic and vector control hairy roots under the PEG condition (data not shown). These results suggested that GmERF75 could improve salinity and exogenous ABA tolerance in soybean.

\section{Discussion}

Transcription factors function as either activators or repressors that up-regulate or down-regulate, respectively, a whole array of target genes, overexpression of which can modulate stress tolerance in plants [32]. Numerous transcription factors has been reported involving in defense against multiple abiotic and biotic stimulus in plants, such as WRKY [33, 34], MYB [35], NAC [36], and ERF [30, 37]. Therefore, the identification and functional analysis of new transcription factor genes is of great importance for understanding the molecular mechanisms of stress tolerance in plants, which may aid efforts to improve crop productivity. ERF transcription factors have been shown to be involved in the response 


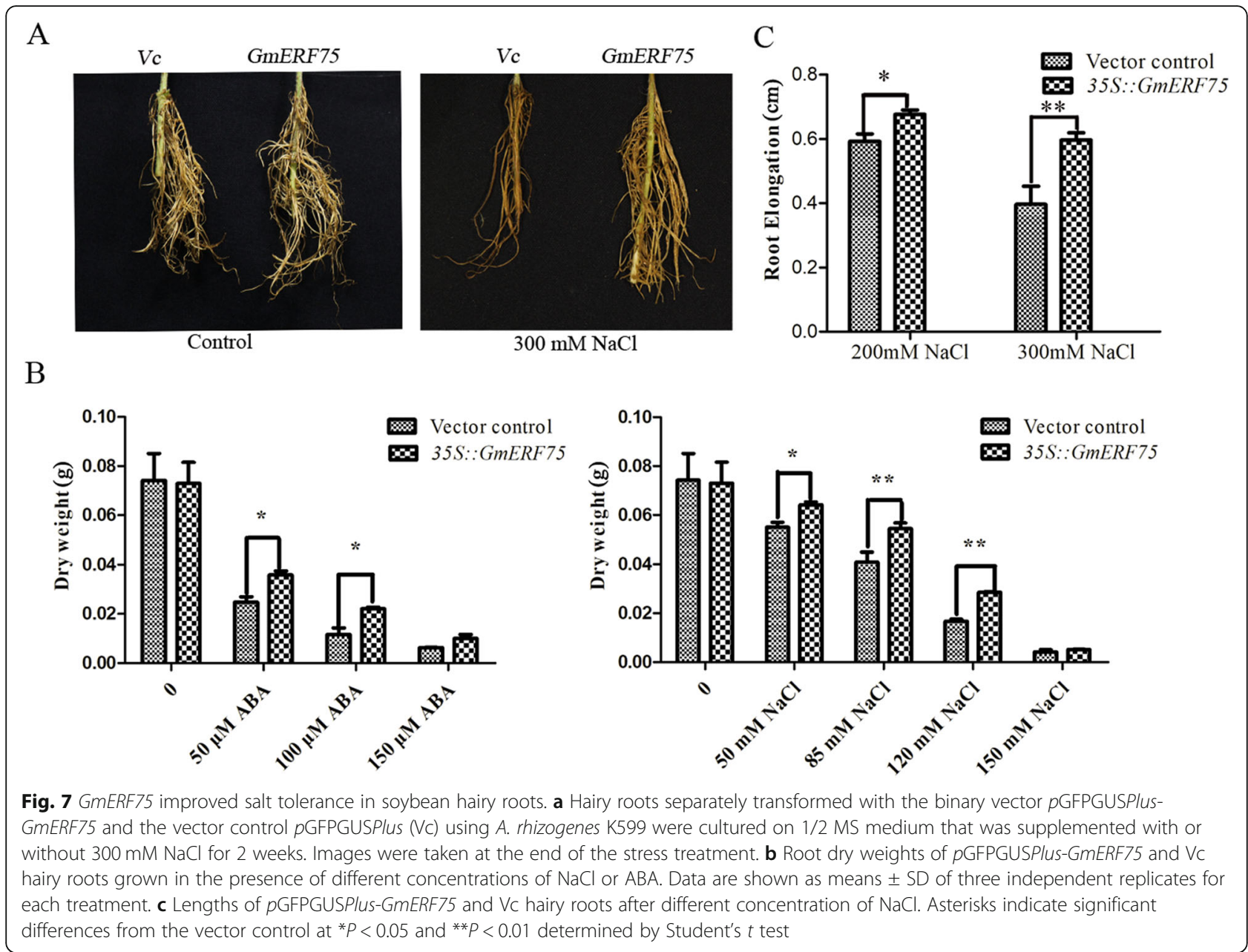

to environmental stresses [5]. In this study, a comprehensive set of 160 soybean ERFs was identified and characterized. To better understand ERF-mediated stress responses, a highly ABA-induced soybean ERF, GmERF75, was isolated and its involvement in stress signal transduction pathways was investigated.

\section{GmERF75 may integrate the SA and ET/JA pathways}

The signal transduction pathways under abiotic stress were extremely complicated and complex in higher plants [38]. Hormones signaling transduction pathways were associated with different environmental stresses when plants resist various stresses, such as drought, salt, cold. It has been verified that there is an antagonistic effect between SA and JA pathways and between the JA/ ET and ABA pathways which could precisely regulated the stress-related gene expression [39-41]. Accordingly, the expression levels of some plant defense genes are impacted via multiple signaling pathways during defense responses [42].

It is known that certain ERF transcription factors are targets of different signaling pathways [5]. For example,
ERF1 can be activated rapidly by ET or JA or synergistically activated by both $[43,44]$. AtERF4, which acts as a transcriptional repressor, can be induced by both ET and JA [13, 45]. Meanwhile, the SA signal transduction pathway can act antagonistically with the ET/JA pathway $[46,47]$. However, in this study, the GmERF75 gene could be induced by exogenous SA, JA, and ET, which indicates that the transcription of GmERF75 can be activated by both the SA and JA/ET pathways (Fig. 5) [48]. These results indicate that GmERF75 may integrate signals from the SA and ET/JA pathways but does not contribute to the antagonistic interplay between them during the soybean seedling stage.

The role of GmERF75 in enhancing hypocotyl length Hypocotyl elongation is regulated by a combination of extrinsic and intrinsic signals, including light and plant hormones [49-51]. Plants have evolved a complicated network of photoreceptors and numerous downstream signaling factors that enable them to respond and adapt to the ambient light environment [52]. vonArnim et al. found that Arabidopsis seedlings grown under light 
displayed short hypocotyls and open cotyledons with functional chloroplasts via photomorphogenesis, while dark-grown plants exhibit long hypocotyls and closed cotyledons and develop etioplasts via a process termed etiolation or skotomorphogenesis [53]. It was reported that light is closely related to hypocotyl cell elongation $[54,55]$, and that photoreceptors can modulate downstream transcription factors, such as ELONGATED HYPOCOTYL5 (HY5) [56]. HY5 can indirectly affect the transduction of many hormone signal transduction pathways, such as ABA, ET, and JA [57]. In this study, GmERF75 was mainly expressed in hypocotyls (Additional file 6: Figure S4) and could be induced by exogenous ABA, ET, and JA (Fig. 5), which suggested GmERF75 functions downstream of these hormone signaling pathways. The erf 71 mutants displayed shorter hypocotyls, while the hypocotyls of GmERF75::erf71 lines were not significantly different in length to WT hypocotyls (Additional file 7: Figure S5). These results implied that GmERF75 may participate in the light-photoreceptorHY5-ABA/ET/JA signal transduction pathway to modulate hypocotyl growth. In addition, promoter analysis showed there are six light-responsive cis-elements in the promoter region of GmERF75, which suggested that this gene may be directly regulated by light. Taken together, these results suggested that GmERF75 may regulate hypocotyls elongation through light-related signaling pathways.

\section{GmERF75 may be an essential factor in diverse abiotic signaling pathways}

It is well known that there are complex connections among various hormones and stress signaling pathways in plants, and a single gene may play roles in many different signaling pathways at same time. Overexpression of $J c D R E B 2$, a physic nut AP2/ERF gene, in rice can suppress the expression of some gibberellic acid biosynthetic genes and induce salt tolerance-related genes to regulate salt stress response [58]. AhDREB1 is an important member of the AP2/ERF family in peanut. Arabidopsis plants overexpressing AhDREB1 had higher ABA sensitivity compared with WT and the expression levels of downstream drought stress-related genes were altered, which demonstrated that overexpression of AhDREB1 could improve tolerance to drought by affecting the ABA-dependent pathway [59]. Similarly, transgenic tobacco plants expressing GmERF9 had enhanced tolerance to drought and cold stresses and increased expression levels of PR genes such as PR1 and PR2 [60]. In this study, both transgenic Arabidopsis plants and soybean hairy roots expressing GmERF75 showed high salt stress tolerance and lower ABA sensitivity. These results suggested that GmERF75 may be involved in salt- and ABA-related signaling pathways. Based on these findings, we conclude that GmERF75 encodes a transcription factor that is likely to be an important determinant of osmotic stress signal transduction pathways in Arabidopsis and soybean.

\section{Conclusion}

GmERF75, protein localized in the nucleus, is responsive to multiple abiotic stresses and exogenous hormones. Two independent Arabidopsis mutations of AtERF71, a gene homologous to GmERF75, displayed shorter hypocotyls, and overexpression of GmERF75 in these mutants could rescue the short hypocotyl phenotypes. GmERF75overexpressing Arabidopsis lines showed higher chlorophyll content under drought and salt stress. Overexpressing GmERF75 in soybean hairy roots improved root growth under exogenous ABA and salt stress. GmERF75 is an important plant transcription factor that plays a critical role in enhancing osmotic tolerance in both Arabidopsis and soybean.

\section{Methods \\ Database searches and the chromosomal distribution of ERF genes in the soybean genome}

The whole genome sequence and repeat information for soybean were obtained from the JGI Glyma1.0 annotation (http://www.phytozome.net/index.php) [61]. The gene chip data for soybean were obtained from SoyBase (http:// www.soybase.org/) [62]. The chromosomal distribution was determined using the chromosome locus information from Phytozome. The MapInspect program was used to draw the chromosomal distribution map.

\section{Alignment and phylogenetic analysis}

We used the Pfam [24] (http://pfam.sanger.ac.uk/) and SMART databases [25] (http://smart.embl-heidelberg.de/) as references for the identification of 160 non-redundant soybean ERFs (Additional file 1: Table S1). Amino acid sequence alignments were performed using ClustalX and were manually corrected. Neighbor-joining method was used to construct the phylogenetic tree of soybean ERFs by MEGA 5.1 [26].

\section{Expression profiles and gene structure analysis}

Expression analysis was conducted using soybean GeneChip expression data for different tissues and developmental stages. The genomic DNA sequences and corresponding coding sequences of the 12 soybean ERF genes were submitted to the Gene Structure Display Server (GSDS) website (http://gsds.cbi.pku.edu.cn/) to visualize the gene structures [63]. The conserved motifs were analyzed using multiple EM for motif elicitation (MEME) software. The sequences were aligned using DNAMAN software. 
Protein domain and homology modeling

The amino acid sequences of the 12 Group VII ERF genes were submitted to the Protein Fold Recognition Server (PHYRE2) (http://www.sbg.bio.ic.ac.uk/phyre2/ $\mathrm{html} /$ page.cgi?id=index) for structural homology modeling. DOG 2.0 was used to draw the protein domains.

\section{Plant materials and stress treatments}

Soybean seedlings (Glycine max cv. Tiefeng 8) grown in soil at $25^{\circ} \mathrm{C}$ for 14 days were subjected to various abiotic stress and exogenous hormone treatments. To investigate the effects of exogenous ABA on ERF transcript family, the soybean seedlings were incubated in $100 \mu \mathrm{M}$ ABA for $0,0.5,1,2,4,8$, or $12 \mathrm{~h}$ [64]. To investigate the effects of abiotic stresses on ERF transcript family, seedlings were subjected to stress for $0,0.5,1,2,5,12$, or 24 h. For rapid induction of drought stress, seedlings were exposed to air on filter paper [65]. For cold stress, seedlings were placed in a $4{ }^{\circ} \mathrm{C}$ chamber [66]. For hightemperature treatment, seedlings were placed in a $42{ }^{\circ} \mathrm{C}$ oven, and for salt stress, seedlings were incubated in $200 \mathrm{mM} \mathrm{NaCl}$ [35]. To investigate the effects of the exogenous hormones SA and JA on physiological and molecular responses, seedlings were incubated in $50 \mu \mathrm{M}$ SA and $50 \mu \mathrm{M} \mathrm{JA}$, respectively for $0,0.5,1,2,5,12$, or $24 \mathrm{~h}$. To evaluate the response to ET, seedlings were placed in a sealed plastic box with a concentration of $200 \mu \mathrm{l} 1^{-1}$ by injection of ethylene for $0,0.5,1,2,5,12$, or $24 \mathrm{~h}[20,67,68]$. For each treatment, 42 individuals were distributed to three groups for three sample replicates. Hypocotyls and roots of two individuals were collected as a sample at each time point, frozen immediately in liquid nitrogen, and stored at $-80^{\circ} \mathrm{C}$ for RNA extraction. There are three repetitions at each time point of each treatment.

\section{RNA extraction, semi-qPCR, and qRT-PCR}

Trizol reagent was used to extract total RNA of the hypocotyls and roots according to the protocol (TIANGEN, China). After treated by DNase I, total RNA was used to synthesize cDNA using PrimeScript First-Strand cDNA Synthesis Kit (TaKaRa, Japan). Semi-qPCR was conducted to investigate the expression pattern of GmERF75 in different soybean plant tissues. RNA was extracted from hypocotyls, roots, stems, and leaves of soybean seedlings. Parallel reactions amplifying actin were performed to normalize the expression levels. qRTPCR was used to analyze the expression patterns of several soybean ERF genes in response to various abiotic stresses and exogenous hormones. qRT-PCR analysis of soybean ERFs was performed using the SYBR Premix Ex $\mathrm{Taq}^{\mathrm{Tm}}$ kit (TaKaRa, Japan) according to the manufacturer's protocol. The expression patterns were analyzed using ABI Prism 7500 sequence detection system
(ThermoFisher Scientific, USA) as previously described $[69,70]$. The soybean ERF gene primers for qRT-PCR were designed to anneal to regions outside the conserved AP2/ERF domain using Primer Premier 5.0 software, and soybean Actin (U60506) [71] was used as an internal control for normalizing the amount of template cDNA. The primers used for qRT-PCR are listed in Additional file 9: Table S3.

\section{Cloning of GmERF75}

The full-length ORF of GmERF75 was amplified from soybean cDNA using the primers $5^{\prime}$-ATGGCGAACGCAGC TGAAGTTT-3' and 5' ${ }^{\prime}$-TCACACCGCCACGAGCG-3'. The PCR product was cloned into the $p$ EASY-T1 vector (TransGen, China).

\section{Subcellular localization assay}

To investigate the biological activity of the putative NLSs, the full-length cDNA sequence of GmERF75 was fused to the N-terminus of the humanized green fluorescent protein $(h G F P)$ gene under the control of the double Cauliflower Mosaic Virus $(2 \times \mathrm{CaMV}) 35 \mathrm{~S}$ promoter. The recombinant plasmid and control plasmid ( $h$ GFP vector) were bombarded into living onion epidermal cells. Visualization of $h G F P$ expression in the onion epidermal cells was performed as described previously [20,33].

\section{Generation of transgenic Arabidopsis and stress treatments}

The coding sequence of GmERF75 was amplified using the primers 5'-TGATTACGCCAAGCTTATGGCGAA CGCAGCTGAAGTTT-3' and 5'-CCGGGGATCCTCTA GACACCGCCACGAGCG-3' and cloned into pBI121 under the control of the CaMV $35 \mathrm{~S}$ promoter to generate the 35S::GmERF75 construct. The construct was confirmed by sequencing and then transformed into WT Arabidopsis plants (Col-0) using the vacuum infiltration method [72, 73]. The transgenic Arabidopsis seeds were screened and T3 seeds of two transgenic lines were used for further phenotypic analysis.

For phenotype analysis, GmERF75 overexpression, erf71 mutant, and WT Arabidopsis seedlings at the two-leaf stage were transferred to MS medium containing 6\% PEG, $75 \mathrm{mM} \mathrm{NaCl}$, or placed in dark. For each treatment, fifteen to twenty-five individuals of each line were used to count the hypocotyl length. Three independent biological replicates were performed for each treatment.

To test the resistance of salt and drought in late stage, 72 Arabidopsis seedlings of GmERF75 overexpression, erf71 mutant, and WT, respectively, were transferred into soil for normally growth after germinating on the MS medium. For each line, all the seedlings were divided into 12 pots, each of which planted 6 seedlings per pot. 
Three-week-old seedlings were supplied with $250 \mathrm{mM}$ $\mathrm{NaCl} 2$ weeks for salt treatment. Three-week-old seedlings normally grown in soil were not watered for drought treatment. A week later, different phenotypes were observed. The Arabidopsis plants were re-watered and recovered for 1 week, and the leaves of each line were collected. Plants normally watered were used as a control. Three independent replicates were performed for each treatment. In order to quantify the phenotype of Arabidopsis response to salt and drought, the chlorophyll content of each line were determined according to the protocol (Cominbio, China). Take $0.1 \mathrm{~g}$ of Arabidopsis leaves of each line and wash them with distilled water. Add $1 \mathrm{~mL}$ of $80 \%$ acetone, mix well and leaching overnight until the leaves are completely white. Add $80 \%$ acetone to $1 \mathrm{~mL}$ cuvette and zero the cuvette. The absorbance values of the samples at $663 \mathrm{~nm}$ and $645 \mathrm{~nm}$ were measured and recorded as $A_{663}$ and $\mathrm{A}_{645}$. Total chlorophyll content $(\mathrm{mg} / \mathrm{g} F W)=\left(20.21^{*}\right.$ $\left.\mathrm{A}_{645}+8.02 * \mathrm{~A}_{663}\right) * 1 \mathrm{~mL} / 0.05 \mathrm{~g} / 1000$.

\section{Soybean hairy root induction and stress treatments}

Seedling growth, rooting, hairy root induction, and hairy root transformation were performed as described by Chen et al. [74, 75]. Chlorine gas-sterilized soybean seeds were germinated in B5 medium. The cotyledons of 4-day-old seedlings as explant were harvested and wounded with a scalpel with K599 carrying the $p$ GFPGUSPlusGmERF75 binary vector for 5 days growth, which was used to transform Superroot-derived $L$. corniculatus plants for about 11 days to observe the hairy roots. The positive transgenic hairy roots were verified via fluorescence GFP. Then a total of 256 GFP-positive $\left(\mathrm{GFP}^{+}\right)$hairy roots were cultured on $1 / 2$ MS medium that was supplemented with $50,85,120$ or $150 \mathrm{mM} \mathrm{NaCl}$, or 50,100 , or $150 \mu \mathrm{M} \mathrm{ABA}$ and incubated at $24^{\circ} \mathrm{C}$ under a $16 / 8 \mathrm{~h}$ light/dark cycle for 2 weeks. After $24 \mathrm{~h}$ incubation at $105^{\circ} \mathrm{C}$, the dry weight increment (30 roots per unit) was calculated and recorded.

The cotyledonary leaf nodes of 45 soybean seedlings (Glycine $\max \mathrm{cv}$. Tiefeng 8) grown in vermiculite at $25^{\circ} \mathrm{C}$ for 7 days were infected by $p$ GFPGUSPlusGmERF75 and vector control. After growing in the soil for about 20 days, the hairy roots will sprout out. The transgenic hairy roots and the control were supplied with different concentrations of $\mathrm{NaCl}$ treatment for 1 week then the root elongation was measured. Three independent replicates were performed for each treatment.

\section{Statistical analysis}

For experiments with single time point, three biological repetitions were performed. For experiment with multiple time points, three independent biological repetitions and three technical repetitions were performed. The data was shown as the means \pm SD of all of the replicates. Asterisks indicate significant difference or extremely significant difference from the control at ${ }^{*} P<0.05$ or ${ }^{* *} P<0.01$, which was determined by Student's $t$ test.

\section{Supplementary information}

Supplementary information accompanies this paper at https://doi.org/10. 1186/s12870-019-2066-6.

Additional file 1: Table S1. Genetic information for soybean ERFs.

Additional file 2: Figure S1. Analysis of soybean ERF expression in different organs and developmental stages. Normalized expression data for the soybean ERFs were collected from SoyBase (http://www.soybase. org/) (Additional file 3: Table S2). The expression levels (vertical coordinates) are reported in transcripts per million (TPM). The different tissues and developmental stages are shown under the horizontal ordinate. The different colors indicate the expression levels of soybean ERFs.

Additional file 3: Table S2. Expression data during different organs and development periods of soybean ERFs.

Additional file 4: Figure S2. Protein domains in the 12 soybean ERF proteins. DOG 2.0 was used to draw the domains in each protein. The conserved AP2/ERF domain is indicated by blue boxes.

Additional file 5: Figure S3. Intron-exon structures of the 12 soybean ERF genes. The diagrams of intron-exon structure were generated using the GSDS online tool. The exons, introns, and untranslated regions (UTRs) are indicated by yellow boxes, black lines, and blue boxes, respectively.

Additional file 6: Figure S4. GmERF75 expression in specific tissues of soybean plants under normal growth conditions. RNA was extracted from hypocotyls, roots, stems, and leaves of soybean seedlings. Parallel reactions amplifying Actin were performed to normalize the expression levels.

Additional file 7: Figure S5. Nucleotide and deduced amino acid sequences of the GmERF75 gene. Untranslated regions (UTRs) and intron sequences are indicated by lowercase letters. The deduced amino acid sequence is shown below the DNA sequence. The AP2/ERF domain is underlined. Basic amino acid regions that potentially act as nuclear localization signals are outlined by boxes, and an acidic amino acid region that may act as a transcriptional activation domain is shown in bold italics. A potential $\mathrm{N}$-linked glycosylation site is indicated by a dotted line.

Additional file 8: Figure S6. GmERF75 rescued the short hypocotyl length phenotype of two erf71 mutants. (A) The erf71 mutants displayed shorter hypocotyls than the WT. (B) Overexpression of GMERF75 in the mutants partially rescued the short hypocotyl length phenotype. The histogram on the right shows the distribution of hypocotyl lengths for at least 30 seedlings.

Additional file 9: Table S3. Primers used for qRT-PCR of soybean ERFs in Group VII.

\section{Abbreviations}

ABA: Abscisic acid; ACC: 1-aminocyclopropane-l-carboxylic acid; AD: Activation domain; AP2: APETALA2; CaMV: Cauliflower mosaic virus; DPBF: DO promoter-binding factors; DRE/CRT: Dehydration-responsive element/C-repeat; EREBP: Ethylene-responsive element binding protein; ERF: Ethylene-responsive factor; ET: Ethylene; GFP: Green fluorescent protein; GUS: $\beta$-glucuronidase; JA: Jasmonic acid; qRT-PCR: quantitative real-time PCR; RT-PCR: Reverse transcription PCR; SA: Salicylic acid; WT: Wild type

\section{Acknowledgments}

We thank Dr. Ryo Akashi for providing the Superroot culture of L. corniculatus; Peter Gresshoff for providing A. rhizogenes strain K599 and the binary vector pGFPGUSPlus; and Dr. Wen-Sheng Hou for providing plant material preparation, technical assistance and soybean seeds. 


\section{Authors' contributions}

ZSX coordinated the project, conceived and designed experiments, and edited the manuscript; MJZ and LJY conducted bioinformatics analysis, performed experiments and wrote the first draft; $\mathrm{MJ}$ and $\mathrm{YL}$ conducted bioinformatics analysis; JCZ, JHL, and JDF contributed valuable discussion and substantively revised it; $\mathrm{MC}$ provided analytical tools and analyzed the data; YZM coordinated the project and edited the manuscript. All authors have read and approved the final manuscript.

\section{Funding}

The design and data collection of this study was supported by the National Natural Science Foundation of China (31871624). Analysis and interpretation of data in this research was supported by the National Transgenic Key Project of the Ministry of Agriculture (2018ZX0800909B). The manuscript was written with financial support from the Talents Introduced Fund of Anhui Science and Technology University (NXYJ201604).

\section{Availability of data and materials}

The datasets using for the present study are available in the JGI Glyma1.0 repository, https://phytozome.jgi.doe.gov/pz/portal.html.

\section{Ethics approval and consent to participate}

Not applicable.

\section{Consent for publication}

Not applicable.

\section{Competing interests}

The authors declare that they have no competing interests.

\section{Author details}

'Institute of Crop Sciences, Chinese Academy of Agricultural Sciences (CAAS)/National Key Facility for Crop Gene Resources and Genetic Improvement, Key Laboratory of Biology and Genetic Improvement of Triticeae Crops, Ministry of Agriculture, Beijing 100081, China. ${ }^{2}$ Faculty of Agronomy, Jilin Agricultural University, Changchun 130118, China. ${ }^{3}$ Anhui Science and Technology University, Fengyang 233100, China. ${ }^{4}$ College of Agronomy, Qingdao Agricultural University, Qingdao 266109, China.

\section{Received: 29 June 2019 Accepted: 2 October 2019}

\section{Published online: 20 November 2019}

\section{References}

1. Atkinson NJ, Urwin PE. The interaction of plant biotic and abiotic stresses: from genes to the field. J Exp Bot. 2012;63(10):3523-43.

2. Singh KB, Foley RC, Onate-Sanchez L. Transcription factors in plant defense and stress responses. Curr Opin Plant Biol. 2002;5(5):430-6.

3. Qin LP, Wang LQ, Guo Y, Li Y, Umut H, Wang YC. An ERF transcription factor from Tamarix hispida, ThCRF1, can adjust osmotic potential and reactive oxygen species scavenging capability to improve salt tolerance. Plant Sci. 2017;265:154-66.

4. Feng ZJ, He GH, Zheng WJ, Lu PP, Chen M, Gong YM, Ma YZ, Xu ZS. Foxtail millet NF-Y families: genome-wide survey and evolution analyses identified two functional genes important in abiotic stresses. Front Plant Sci. 2015;6:1142.

5. Xu ZS, Chen M, Li LC, Ma YZ. Functions of the ERF transcription factor family in plants. Botany. 2008;86(9):969-77.

6. Licausi F, Ohme-Takagi M, Perata P. APETALA2/ethylene responsive factor (AP2/ERF) transcription factors: mediators of stress responses and developmental programs. New Phytol. 2013;199(3):639-49.

7. Nakano T, Suzuki K, Fujimura T, Shinshi H. Genome-wide analysis of the ERF gene family in Arabidopsis and rice. Plant Physiol. 2006;140(2):411-32.

8. Li A, Yu X, Cao BB, Peng LX, Gao Y, Feng T, Li H, Ren ZY. LkAP2L2, an AP2/ ERF transcription factor gene of Larix kaempferi, with pleiotropic roles in plant branch and seed development. Russ J Genet. 2017;53(12):1335-42.

9. Sun WQ, Gao DW, Xiong Y, Tang XX, Xiao XF, Wang CR, Yu SB. Hairy leaf 6, an AP2/ERF transcription factor, interacts with OsWOX3B and regulates Trichome formation in Rice. Mol Plant. 2017;10(11):1417-33.

10. Allen MD, Yamasaki K, Ohme-Takagi M, Tateno M, Suzuki M. A novel mode of DNA recognition by a beta-sheet revealed by the solution structure of the GCC-box binding domain in complex with DNA. EMBO J. 1998;17(18): 5484-96.
11. Welsch R, Maass D, Voegel T, DellaPenna D, Beyer P. Transcription factor RAP2.2 and its interacting partner SINAT2: stable elements in the carotenogenesis of Arabidopsis leaves. Plant Physiol. 2007;145(3):1073-85.

12. Gasch P, Fundinger M, Muller JT, Lee T, Bailey-Serres J, Mustroph A. Redundant ERF-VII transcription factors bind to an evolutionarily conserved cis-motif to regulate hypoxia-responsive gene expression in Arabidopsis. Plant Cell. 2016;28(1):160-80.

13. Yang Z, Tian LN, Latoszek-Green M, Brown D, Wu KQ. Arabidopsis ERF4 is a transcriptional repressor capable of modulating ethylene and abscisic acid responses. Plant Mol Biol. 2005;58(4):585-96.

14. Sun $X H$, Yu G, Li JT, Liu JL, Wang XL, Zhu GL, Zhang XH, Pan HY. AcERF2, an ethylene-responsive factor of Atriplex canescens, positively modulates osmotic and disease resistance in Arabidopsis thaliana. Plant Sci. 2018;274 32-43.

15. Wan LY, WU YS, Huang JQ, Dai XF, Lei Y, Yan LY, Jiang HF, Zhang JC, Varshney RK, Liao BS. Identification of ERF genes in peanuts and functional analysis of AhERF008 and AhERF019 in abiotic stress response. Funct Integr Genomic. 2014;14(3):467-77.

16. Najafi S, Sorkheh K, Nasernakhaei F. Characterization of the APETALA2/ ethylene-responsive factor (AP2/ERF) transcription factor family in sunflower. Sci Rep-Uk. 2018;8(1):11576.

17. Xie XL, Xia XJ, Kuang S, Zhang XL, Yin XR, Yu JQ, Chen KS. A novel ethylene responsive factor CitERF13 plays a role in photosynthesis regulation. Plant Sci. 2017;256:112-9.

18. Tian ZD, He Q, Wang HX, Liu Y, Zhang Y, Shao F, Xie CH. The potato ERF transcription factor StERF3 negatively regulates resistance to Phytophthora infestans and salt tolerance in potato. Plant Cell Physiol. 2015;56(5):992-1005.

19. Giuntoli B, Shukla V, Maggiorelli F, Giorgi FM, Lombardi L, Perata P, Licausi F. Age-dependent regulation of ERF-VII transcription factor activity in Arabidopsis thaliana. Plant Cell Environ. 2017:40(10):2333-46.

20. Xu ZS, Xia LQ, Chen M, Cheng XG, Zhang RY, Li LC, Zhao YX, Lu Y, Ni ZY, $L i u L$, et al. Isolation and molecular characterization of the Triticum aestivum L. ethylene-responsive factor 1 (TaERF1) that increases multiple stress tolerance. Plant Mol Biol. 2007;65(6):719-32.

21. Xing LP, Di ZC, Yang WW, Liu JQ, Li MN, Wang XJ, Cui CF, Wang XY, Wang $X E$, Zhang RQ, et al. Overexpression of ERF1-V from Haynaldia villosa can enhance the resistance of wheat to powdery mildew and increase the tolerance to salt and drought stresses. Front Plant Sci. 2017;8:1948.

22. Zhu XL, Qi L, Liu X, Cai SB, Xu HJ, Huang RF, Li JR, Wei XN, Zhang ZY. The wheat ethylene response factor transcription factor PATHOGEN- INDUCED ERF1 mediates host responses to both the Necrotrophic pathogen Rhizoctonia cerealis and freezing stresses. Plant Physiol. 2014;164(3):1499-514.

23. Jin $Y$, Pan WY, Zheng XF, Cheng X, Liu MM, Ma H, Ge XC. OsERF101, an ERF family transcription factor, regulates drought stress response in reproductive tissues. Plant Mol Biol. 2018;98(1-2):51-65.

24. Finn RD, Coggill P, Eberhardt RY, Eddy SR, Mistry J, Mitchell AL, Potter SC, Punta M, Qureshi M, Sangrador-Vegas A, et al. The Pfam protein families database: towards a more sustainable future. Nucleic Acids Res. 2016;44(D1): D279-85.

25. Schultz J, Milpetz F, Bork P, Ponting CP. SMART, a simple modular architecture research tool: identification of signaling domains. P Natl Acad Sci USA. 1998:95(11):5857-64.

26. Tamura K, Peterson D, Peterson N, Stecher G, Nei M, Kumar S. MEGA5: molecular evolutionary genetics analysis using maximum likelihood, evolutionary distance, and maximum parsimony methods. Mol Biol Evol. 2011;28(10):2731-9.

27. Schroeder JI, Kwak JM, Allen GJ. Guard cell abscisic acid signalling and engineering drought hardiness in plants. Nature. 2001;410(6826):327-30.

28. Xiong LM, Schumaker KS, Zhu JK. Cell signaling during cold, drought, and salt stress. Plant Cell. 2002;14:S165-83.

29. Seki M, Ishida J, Narusaka M, Fujita M, Nanjo T, Umezawa T, Kamiya A, Nakajima M, Enju A, Sakurai T, et al. Monitoring the expression pattern of around 7,000 Arabidopsis genes under ABA treatments using a full-length cDNA microarray. Funct Integr Genomics. 2002;2(6):282-91.

30. Xu ZS, Chen M, Li LC, Ma YZ. Functions and application of the AP2/ERF transcription factor family in crop improvement. J Integr Plant Biol. 2011; 53(7):570-85.

31. Wang X, Jing YJ, Zhang BC, Zhou YH, Lin RC. Glycosyltransferase-like protein ABI8/ELD1/KOB1 promotes Arabidopsis hypocotyl elongation through regulating cellulose biosynthesis. Plant Cell Environ. 2015;38(3):411-22. 
32. Ohama N, Sato H, Shinozaki K, Yamaguchi-Shinozaki K. Transcriptional regulatory network of plant heat stress response. Trends Plant Sci. 2017; 22(1):53-65.

33. He GH, Xu JY, Wang YX, Liu JM, Li PS, Chen M, Ma YZ, Xu ZS. Droughtresponsive WRKY transcription factor genes TaWRKY1 and TaWRKY33 from wheat confer drought and/or heat resistance in Arabidopsis. BMC Plant Biol. 2016;16(1):116

34. Wang CT, Ru JN, Liu YW, Li M, Zhao D, Yang JF, Fu JD, Xu ZS. Maize WRKY Transcription Factor ZmWRKY106 Confers Drought and Heat Tolerance in Transgenic Plants. Int J Mol Sci. 2018;19(10):3046.

35. Du YT, Zhao MJ, Wang CT, Gao Y, Wang YX, Liu YW, Chen M, Chen J, Zhou $Y B, X u Z S$, et al. Identification and characterization of GmMYB118 responses to drought and salt stress. BMC Plant Biol. 2018;18(1):320.

36. Huysmans M, Buono RA, Skorzinski N, Radio MC, De Winter F, Parizot B, Mertens J, Karimi M, Fendrych M, Nowack MK. NAC transcription factors ANAC087 and ANAC046 control distinct aspects of programmed cell death in the Arabidopsis Columella and lateral root cap. Plant Cell. 2018;30(9): 2197-213.

37. Vogel MO, Moore M, Konig K, Pecher P, Alsharafa K, Lee J, Dietz KJ. Fast retrograde signaling in response to high light involves metabolite export, MITOGEN-ACTIVATED PROTEIN KINASE6, and AP2/ERF transcription factors in Arabidopsis. Plant Cell. 2014;26(3):1151-65.

38. McGrath KC, Dombrecht B, Manners JM, Schenk PM, Edgar CI, Maclean DJ, Scheible WR, Udvardi MK, Kazan K. Repressor- and activator-type ethylene response factors functioning in jasmonate signaling and disease resistance identified via a genome-wide screen of Arabidopsis transcription factor gene expression. Plant Physiol. 2005;139(2):949-59.

39. Anderson JP, Badruzsaufari E, Schenk PM, Manners JM, Desmond OJ, Ehlert C, Maclean DJ, Ebert PR, Kazan K. Antagonistic interaction between abscisic acid and jasmonate-ethylene signaling pathways modulates defense gene expression and disease resistance in Arabidopsis. Plant Cell. 2004;16(12):3460-79.

40. Takahashi H, Kanayama Y, Zheng MS, Kusano T, Hase S, Ikegami M, Shah J. Antagonistic interactions between the SA and JA signaling pathways in Arabidopsis modulate expression of defense genes and gene-for-gene resistance to cucumber mosaic virus. Plant Cell Physiol. 2004;45(6):803-9.

41. Kariola T, Brader G, Li J, Palva ET. Chlorophyllase 1, a damage control enzyme, affects the balance between defense pathways in plants. Plant Cell. 2005;17(1):282-94.

42. Shahnejat-Bushehri S, Tarkowska D, Sakuraba Y, Balazadeh S. Arabidopsis NAC transcription factor JUB1 regulates GA/BR metabolism and signalling. Nat Plants. 2016;2(3):16013.

43. Berrocal-Lobo M, Molina A. Ethylene response factor 1 mediates Arabidopsis resistance to the soilborne fungus Fusarium oxysporum. Mol Plant Microbe Interact. 2004;17(7):763-70.

44. Lorenzo O, Piqueras R, Sanchez-Serrano JJ, Solano R. ETHYLENE RESPONSE FACTOR1 integrates signals from ethylene and jasmonate pathways in plant defense. Plant Cell. 2003:15(1):165-78.

45. Brown RL, Kazan K, McGrath KC, Maclean DJ, Manners JM. A role for the GCC-box in jasmonate-mediated activation of the PDF1.2 gene of Arabidopsis. Plant Physiol. 2003;132(2):1020-32.

46. Zhao YF, Thilmony R, Bender CL, Schaller A, He SY, Howe GA. Virulence systems of Pseudomonas syringae pv. Tomato promote bacterial speck disease in tomato by targeting the jasmonate signaling pathway. Plant J. 2003;36(4):485-99.

47. Glazebrook J, Chen WJ, Estes B, Chang HS, Nawrath C, Metraux JP, Zhu T, Katagiri F. Topology of the network integrating salicylate and jasmonate signal transduction derived from global expression phenotyping. Plant J. 2003:34(2):217-28

48. Fischer $U$, Droge-Laser $W$. Overexpression of NtERF5, a new member of the tobacco ethylene response transcription factor family enhances resistance to tobacco mosaic virus. Mol Plant Microbe Interact. 2004;17(10):1162-71.

49. Van de Poel B, Smet D, Van Der Straeten D. Ethylene and hormonal cross talk in vegetative growth and development. Plant Physiol. 2015;169(1):61-72.

50. Vandenbussche F, Vancompernolle B, Rieu I, Ahmad M, Phillips A, Moritz T, Hedden P, Van Der Straeten D. Ethylene-induced Arabidopsis hypocotyl elongation is dependent on but not mediated by gibberellins. J Exp Bot. 2007;58(15-16):4269-81.

51. Vandenbussche F, Vaseva I, Vissenberg K, Van Der Straeten D. Ethylene in vegetative development: a tale with a riddle. New Phytol. 2012;194(4):895-909.

52. Chen $\mathrm{M}$, Chory J, Fankhauser $\mathrm{C}$. Light signal transduction in higher plants. Annu Rev Genet. 2004;38:87-117.
53. vonArnim A, Deng XW. Light control of seedling development. Annu Rev Plant Phys. 1996:47:215-43.

54. Chaiwanon J, Wang WF, Zhu JY, Oh E, Wang ZY. Information integration and communication in plant growth regulation. Cell. 2016;164(6):1257-68.

55. de Wit M, Galvao VC, Fankhauser C. Light-Mediated Hormonal Regulation of Plant Growth and Development. Annu Rev Plant Biol. 2016;67:513-37.

56. Luo XM, Lin WH, Zhu SW, Zhu JY, Sun Y, Fan XY, Cheng ML, Hao YQ, Oh E, Tian MM, et al. Integration of light- and Brassinosteroid-signaling pathways by a GATA transcription factor in Arabidopsis. Dev Cell. 2010;19(6):872-83.

57. Lau OS, Deng XW. Plant hormone signaling lightens up: integrators of light and hormones. Curr Opin Plant Biol. 2010;13(5):571-7.

58. Tang YH, Liu K, Zhang J, Li XL, Xu KD, Zhang Y, Qi J, Yu DS, Wang J, Li CW. JCDREB2, a physic nut AP2/ERF gene, alters plant growth and salinity stress responses in transgenic Rice. Front Plant Sci. 2017:8:306.

59. Zhang B, Su L, Hu B, Li L. Expression of AhDREB1, an AP2/ERF Transcription Factor Gene from Peanut, Is Affected by Histone Acetylation and Increases Abscisic Acid Sensitivity and Tolerance to Osmotic Stress in Arabidopsis. Int J Mol Sci. 2018;19(5):1441.

60. Zhai Y, Shao SL, Sha W, Zhao Y, Zhang J, Ren WW, Zhang C. Overexpression of soybean GmERF9 enhances the tolerance to drought and cold in the transgenic tobacco. Plant Cell Tiss Org. 2017;128(3):607-18.

61. Goodstein DM, Shu S, Howson R, Neupane R, Hayes RD, Fazo J, Mitros T, Dirks W, Hellsten U, Putnam N, et al. Phytozome: a comparative platform for green plant genomics. Nucleic Acids Res. 2012;40(Database issue):D1178-86.

62. Grant D, Nelson RT, Cannon SB, Shoemaker RC. SoyBase, the USDA-ARS soybean genetics and genomics database. Nucleic Acids Res. 2010;38:D843-6.

63. Guo AY, Zhu QH, Chen X, Luo JC. GSDS: a gene structure display server. Hereditas (Beijing). 2007;29(8):1023-6.

64. Liu K, Yang Q, Yang T, Wu Y, Wang G, Yang F, Wang R, Lin X, Li G. Development of agrobacterium-mediated transient expression system in Caragana intermedia and characterization of CiDREB1C in stress response. BMC Plant Biol. 2019;19(1):237.

65. Li B, Liu Y, Cui XY, Fu JD, Zhou YB, Zheng WJ, Lan JH, Jin LG, Chen M, Ma $Y Z$, et al. Genome-wide characterization and expression analysis of soybean TGA transcription factors identified a novel TGA gene involved in drought and salt tolerance. Front Plant Sci. 2019;10:549.

66. Yamaguchi-Shinozaki K, Shinozaki K. Transcriptional regulatory networks in cellular responses and tolerance to dehydration and cold stresses. Annu Rev Plant Biol. 2006;57:781-803.

67. Li W, Ma M, Feng Y, Li H, Wang Y, Ma Y, Li M, An F, Guo H. ElN2-directed translational regulation of ethylene signaling in Arabidopsis. Cell. 2015; 163(3):670-83.

68. Qiao H, Shen Z, Huang SS, Schmitz RJ, Urich MA, Briggs SP, Ecker JR. Processing and subcellular trafficking of ER-tethered EIN2 control response to ethylene gas. Science. 2012;338(6105):390-3.

69. Li PS, Yu TF, He GH, Chen M, Zhou YB, Chai SC, Xu ZS, Ma YZ. Genomewide analysis of the Hsf family in soybean and functional identification of GmHsf-34 involvement in drought and heat stresses. BMC Genomics. 2014; 15:1009

70. Li ZY, Xu ZS, He GY, Yang GX, Chen M, Li LC, Ma YZ. A mutation in Arabidopsis BSK5 encoding a brassinosteroid-signaling kinase protein affects responses to salinity and abscisic acid. Biochem Bioph Res Co. 2012;426(4): $522-7$.

71. Moniz de Sa M, Drouin G. Phylogeny and substitution rates of angiosperm actin genes. Mol Biol Evol. 1996:13(9):1198-212.

72. Bechtold N, Pelletier G. In planta agrobacterium-mediated transformation of adult Arabidopsis thaliana plants by vacuum infiltration. Methods Mol Biol. 1998;82:259-66

73. Liu P, Xu ZS, Pan-Pan L, Hu D, Chen M, Li LC, Ma YZ. A wheat PI4K gene whose product possesses threonine autophophorylation activity confers tolerance to drought and salt in Arabidopsis. J Exp Bot. 2013;64(10):2915-27.

74. Chen L, Jiang BJ, Wu CX, Sun S, Hou WS, Han TF. The characterization of GmTIP, a root-specific gene from soybean, and the expression analysis of its promoter. Plant Cell Tiss Org. 2015;121(2):259-74.

75. Chen L, Jiang BJ, Wu CX, Sun S, Hou WS, Han TF. GmPRP2 promoter drives root-preferential expression in transgenic Arabidopsis and soybean hairy roots. BMC Plant Biol. 2014;14:245.

\section{Publisher's Note}

Springer Nature remains neutral with regard to jurisdictional claims in published maps and institutional affiliations. 